\title{
Modifications proposed to the concepts of structural protection
}

\begin{abstract}
In this paper we show that the scale of damage in buildings with steel reinforcement, due to direct lightning strikes, is insignificantly small compared to the lightning effects on buildings and structures with no such steel reinforcement. The observed small-scale damages are also confined only to the point of strike. A large number of such cases have been observed in Sri Lanka and Malaysia. The fact that almost all these damaged structures, observed in this study, have a steel reinforcement structure, but not verified to be in compliance with the specifications in IEC 62305-3 (2006) for it to act as down conducting system, prompted us to re-think regarding the specifications. Such specifications are difficult to be achieved in practice and also not applicable to buildings that have been already constructed. The recommendations prevents the engineers from using the steel reinforcement as the down conductor in many cases; a fact that leads to either non-installation of LP systems or adopting non-conventional systems which are convenient to be installed. In both cases, a noteworthy advantage that could have obtained by using the steel reinforcement is lost. Use of the steel reinforcement as the down conducting system, which is integrated to a properly designed airtermination system could have prevented even the small-scale damage occurred at the attachment point. Hence, we strongly recommend revising the relevant recommendations in the standards so that a mass public will be benefited. In the absence of such relaxation of the existing guidelines the manufacturers and proponents of nonconventional lightning protection systems enjoy an undue advantage in promoting their technology.
\end{abstract}

